\title{
Comparación de la fuerza de adhesión a dentina de cementos de autograbado vs grabado total.
}

\section{Comparison of bond strength to dentin of self-etching cements vs total etching.}

\author{
Rogelio Moreno Landa,* Dulce María Saavedra Aguilar,* Ernesto Limón Bernal, \\ Jorge Guerrero Ibarra,
}

\section{RESUMEN}

Los sistemas cementantes han mejorado notablemente, los objetivos que persiguen los nuevos cementos es que la adhesión sea duradera y conseguir siempre que sea posible una interface cerrada con un sellado perfecto. Se han podido desarrollar nuevas técnicas y nuevos materiales de cementación que han ido perfeccionando la unión del material restaurador al diente. En el presente estudio se compara la fuerza de adhesión a dentina de cementos de autograbado y cementos de grabado total para comprobar los efectos positivos en el grabado de la dentina. Para el estudio se utilizaron dos cementos a base de resina (RelyX U200 Clicker 3M y RelyX Ultimate 3M). Se encapsularon 40 molares en acrílico en dos grupos de 20 muestras para la aplicación de dos sistemas cementantes de autograbado (grupo 1) y de grabado total (grupo 2), respectivamente, se desgastaron hasta descubrir la dentina; siguiendo las especificaciones del fabricante se colocó el cemento en cada grupo, y después se sometieron a pruebas de cizalla en una máquina de ensayo universal Instron. La medida expresada en megapascales (MPa) fue: grupo $1=7.5569$ y grupo $2=12.6444$. En este caso fueron analizados dos grupos, tomándose la primera significancia bilateral. Se realiza la prueba en t de Student, con 95\% de intervalo de confianza en la diferencia, demostrando así que el cemento RelyX Ultimate 3M tiene mayor fuerza de adhesión que el cemento RelyX U200 Clicker 3M. Nuestra investigación fue factible y llegamos a nuestro propósito, en el cual comprobamos la mayor adhesión de cementos de grabado total, los cuales son sometidos a un previo tratamiento del diente.

Palabras clave: Adhesión dental, dentina, grabado total, autograbado.

\section{ABSTRACT}

Cementing systems have improved remarkably, the objectives pursued by the new cements is that the adhesion must be durable and always achieve a closed interface with a perfect seal. It has been possible to develop new techniques and new cementation materials that have perfected the bonding of the restorative material to the tooth. In the present study, the adhesion strength to dentin of self-etching cements and total etching cements is compared to verify the positive effects on dentin etching. For the study, two resin-based cements (RelyX U200 Clicker 3M and RelyX Ultimate 3M) were used. 40 molars were encapsulated in acrylic in two groups of 20 samples for the application of two cementitious systems of self-etching (group 1) and total etching (group 2) respectively, they were worn until the dentin was discovered, following the manufacturer's specifications, the cement in each group, and then shear tested on an Instron universal testing machine. The measure expressed in megapascals (MPa) was: group $1=7.5569$ and group $2=12.6444$. In this case, two groups were analyzed, taking the first bilateral significance. The Student t-test is performed, with a 95\% confidence interval in the difference, thus demonstrating that RelyX Ultimate 3M cement has greater bond strength than RelyX U200 Clicker $3 \mathrm{M}$ cement. Our research was feasible and we reached our purpose, which was to verify the greater adhesion of total etching cements, which are subjected to a previous treatment of the tooth.

Keywords: Dental bonding, dentin, total etching, self-etching.

\footnotetext{
* Cirujano Dentista Militar.

¥ Maestro en Ciencias.

§ Sección de Estudios de Postgrado e Investigación, Escuela Superior de Medicina, Instituto Politécnico Nacional. Unidad de Investigación en

Farmacología, Instituto Nacional de Enfermedades Respiratorias Ismael Cosío Villegas, Secretaría de Salud.

" Facultad de Odontología de la Universidad Nacional Autónoma de México.

\| Especialista en Endoperiodontología.

Secretaría de la Defensa Nacional, Escuela Militar de Odontología. Ciudad de México, México.

Recibido: 20 de agosto de 2020. Aceptado: 23 de noviembre de 2021.

Citar como: Moreno LR, Saavedra ADM, Limón BE, Guerrero IJ, Maya MAL. Comparación de la fuerza de adhesión a dentina de cementos de autograbado vs grabado total. Rev ADM. 2022; 79 (1): 20-27. https://dx.doi.org/10.35366/103814
} 


\section{INTRODUCCIÓN}

$\mathrm{E}$ I avance de los sistemas adhesivos se dio a partir del surgimiento del grabado ácido en esmalte propuesto por Michael Buonocore en 1955, que empezó a emplear el ácido fosfórico al 85\%, dando origen a la odontología adhesiva. El siguiente adelanto fue hecho por Bowen en 1962, quien logró desarrollar las resinas compuestas mejorando a sus antecesoras; estudios posteriores ${ }^{1,2}$ demostraron que la creación de áreas microscópicas retentivas en el esmalte permitía que el agente de unión se difundiera, humectara y fijara en ellas al polimerizarlo, generando una zona retentiva entre los sustratos. Fusayama y colaboradores, posteriormente, introdujeron el acondicionamiento de la dentina en conjunto con el grabado del esmalte utilizando ácido fosfórico, llamándole a esta técnica: grabado total. ${ }^{3,4}$

En la actualidad, el desarrollo de los sistemas adhesivos ha estado enfocado a la simplificación de los protocolos de aplicación y al empleo de sistemas más simples como es el surgimiento del sistema autograbado, intentando desplazar el grabado ácido total comúnmente usado, el cual está respaldado por cuantiosa evidencia científica. Falconí y su grupo ${ }^{3}$ demostraron en su estudio que el sistema adhesivo de acondicionamiento con grabado ácido, al aplicar ácido fosfórico al 37\% sobre esmalte y dentina, es el sistema con menores valores de microfiltración reportados y de mayor fiabilidad ante otros sistemas, lo que se explicaría debido al proceso desmineralizante producido tras la colocación del ácido sobre las superficies dentales, y la subsecuente remoción del barrillo dentinario con la exposición de fibra colágena a nivel de dentina, asegurando una mejor interacción del tejido dental, lo que no se logra con los sistemas de autograbado consecuente a su pobre acción sobre el esmalte dental. ${ }^{4}$

El éxito de toda restauración está relacionado con el agente cementante, el cual se denomina como el medio de fijación de dos superficies sólidas según Machi R., el cemento, entonces, es un líquido que fluye y humecta las superficies, penetra en sus irregularidades y llena espacios entre ambas, endureciendo y asegurando el contacto entre esas superficies. ${ }^{5}$

La constante investigación ha favorecido el desarroIlo de nuevos cementos que brinden mayor fuerza de adhesión combinada con una técnica de colocación simple y eficiente, que sean biocompatibles, insolubles al medioambiente bucal, estéticos y con propiedades mecánicas que superen al resto de los cementos. ${ }^{6}$

El cemento es una sustancia sólida cuyo objetivo es unir dos superficies. En odontología, actualmente existen tres tipos de cementos: no adhesivos (fosfato de zinc), de unión química (policarboxilato, ionómero de vidrio, también ionómero de vidrio modificado con resina) y de adhesión micromecánica (cementos con base en dimetacrilato), como los cementos autograbadores. La mayoría de los cementos de resina necesitan un pretratamiento del sustrato dental para promover la adhesión a los tejidos dentarios. Este pretratamiento puede realizarse mediante grabado total con ácido fosfórico, un imprimador y un adhesivo, pero recientemente se han introducido cementos autograbadores que evitan el tradicional grabado total y sus múltiples pasos, y con ello se ha logrado la cementación en un solo paso. ${ }^{7,8}$

Bitter y colaboradores, en $2015,{ }^{9}$ concluyeron que no hubo diferencias significativas en la interfase cementodentina cuando compararon un sistema autograbador con un sistema de grabado total. Como resultado de su investigación, mostraron una capa híbrida homogénea dentro del conducto radicular para ambos sistemas de cementos. Sridhara y colaboradores ${ }^{10}$ compararon el espesor de la capa híbrida entre dos tipos de cementos: Panavia $\mathrm{F}^{\circledR}$ (autoadhesivo) y All Bond $2^{\circledR}$ (grabado total). Concluyeron que con All Bond 2 el espesor de la capa híbrida es mejor y más efectivo, ya que crea entrelazados micromecánicos entre el material adhesivo y la dentina grabada. Por otra parte, Esclassan Noirrit y colaboradores ${ }^{11}$ compararon las técnicas de autograbado y grabado total en cuanto a la formación de capa híbrida. No hallaron diferencias significativas entre estas dos técnicas. Posteriormente, Julio Chávez Lozada y su equipo, ${ }^{12}$ en su estudio, concluyeron que los cementos autoadhesivos evaluados presentaron menor resistencia adhesiva que los cementos resinosos que requieren tratamiento previo del sustrato dentario y que esto debería ser considerado cuando se realiza su selección clínica, sobre todo en preparaciones dentarias poco retentivas. ${ }^{12,13}$

Por lo anterior, es necesario caracterizar y evaluar la dentina, el protocolo de adhesión convencional y los avances actuales de la técnica, de manera que este conocimiento sea usado como base de futuras investigaciones que busquen mejorar el desempeño de los materiales adhesivos. ${ }^{14}$

\section{MATERIAL Y MÉTODOS}

\section{Material}

Caretas de protección.

Curetas Hu-Friedy.

Guantes de látex o vinil. 
Batas de laboratorio.

Acrílico Nictone $35 \mathrm{~g}$.

Monómero $950 \mathrm{~mL}$.

Godetes de plástico.

Envases plásticos para recolectar las muestras.

Pinzas de exploración Hu-Friedy.

Micromotor (Strong 90).

Porta disco metálico y disco de diamante.

Cemento RelyX U200 Clicker 3M.

RelyX Ultimate Clicker 3M.

Ácido grabador.

Adhesivo primer Single Bond ${ }^{\text {TM }}$ Universal.

Lijas número 600 y 120.

Lámpara Bluephase N MC 100-240V.

\section{Métodos}

1. Para la evaluación y comparación se recolectaron 40 dientes (premolares o terceros molares sanos) (Figura 1), con indicación de extracción y que los pacientes tuvieran entre 16 y 40 años de edad, que no tengan más de seis meses de su extracción, los cuales fueron conservados en un recipiente de vidrio cerrado con agua bidestilada para mantener su correcta hidra-

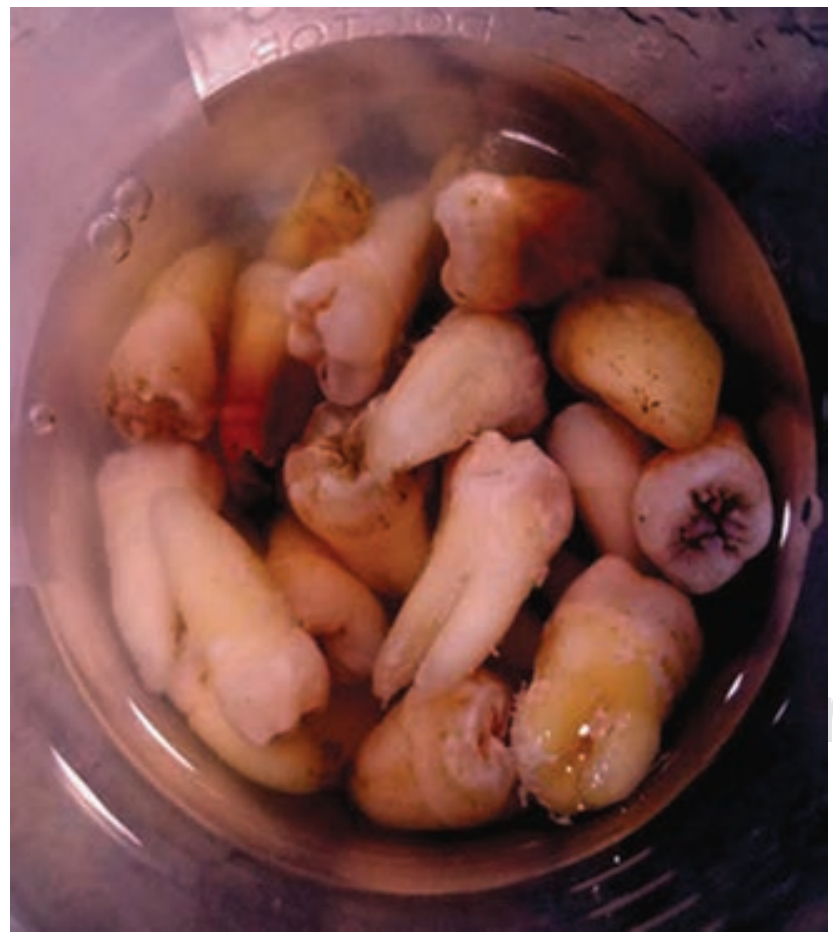

Figura 1: Recolección de 40 dientes.

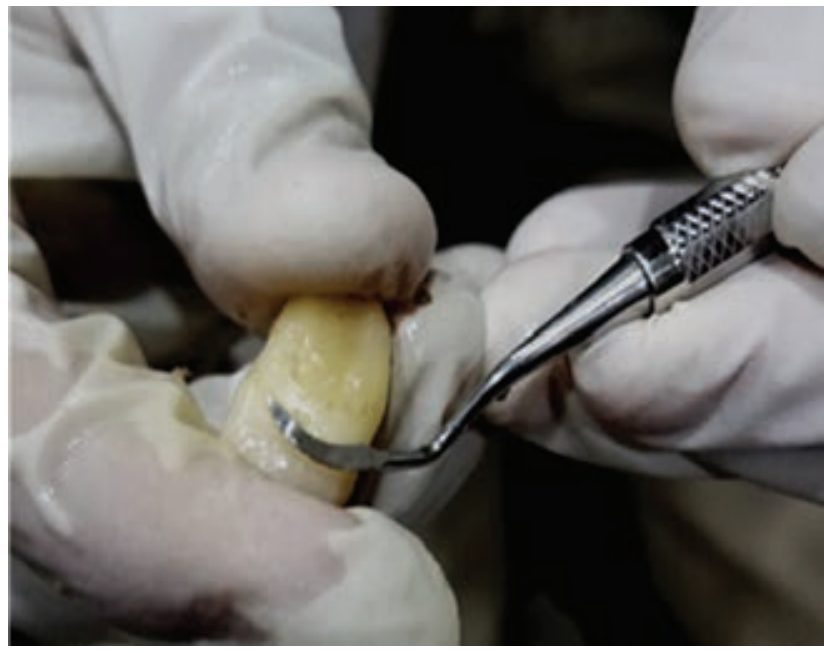

Figura 2: Eliminación de restos orgánicos.

tación, mantenidos a una temperatura ambiente indicado en la norma ISO/TS 11405.

2. Los órganos dentarios se limpiaron con curetas y cepillos profilácticos (Figura 2), para eliminar restos orgánicos que estén adosados en su superficie.

3. Se montaron en acrílico a través de anillos de polipropileno de una pulgada de diámetro interno (Figura 3), mismos que se sometieron a un desgaste de $3 \mathrm{~mm}$ en la máquina de esmerilado y pulido struers con lijas Fandeli de grano 600 y 120 para descubrir dentina.

4. Las muestras se colocaron en un recipiente donde se diferenciaron los dos diferentes grupos.

5. Posteriormente, colocamos el ácido grabador (ácido fosfórico al 37\%) durante 15 segundos en las muestras del grupo A (grabado total), lavamos durante $1 \mathrm{~min}$ y secamos las muestras.

6. Colocamos adhesivo Single Bond Universal a la superficie del diente de acuerdo con las indicaciones del fabricante (Figura 4).

7. Después, con ayuda de aditamentos, se colocó un botón de cemento a cada muestra de acuerdo con las indicaciones del fabricante.

8. Almacenamos durante 24 horas a una temperatura de $37^{\circ} \mathrm{C}$ y una humedad relativa del 95 al $100 \%$ simulando la cavidad oral (Figura 5). ${ }^{15}$

9. Antes de realizar las pruebas de fuerza de adhesión al cizallamiento, calculamos el área de los cilindros de resina (Figura 6), para introducirlos a la computadora. La prueba se realizó en cada espécimen en una máquina universal de pruebas mecánicas (Instron), 
donde colocamos las muestras en el sujetador para que después, con la cizalla, apliquemos la fuerza dirigida hacia la cementación colocada con cemento RelyX U200 Clicker 3M y cemento RelyX Ultimate Clicker 3M, a una velocidad del cabezal de $1 \mathrm{~mm} /$ min hasta el desprendimiento de la resina.

10. Los valores de resistencia obtenidos se expresaron en megapascales (MPa).

\section{RESULTADOS}

En la presente investigación se empleó un formato de «tablero de control», la cual es una herramienta que se utilizó para diagnosticar adecuadamente los resultados que arrojó la máquina Instron a través de la fuerza de resistencia al cizallamiento.

\section{Modelo de calidad en el análisis de resultados}

En el presente estudio se sometieron 40 muestras divididas en dos grupos de 20 especímenes cada uno a pruebas de fuerza de adhesión al cizallamiento, obteniendo los resultados mostrados en la Tabla 1.

Aplicando la estadística de grupo se representan los valores descriptivos de la media y desviación estándar en MPa, obtenidos de la máquina (Instron) (Tabla 2).

Los materiales comprados fueron dos tipos de cemento de autograbado y grabado total; el primero RelyX U200 y el segundo RelyX Ultimate.

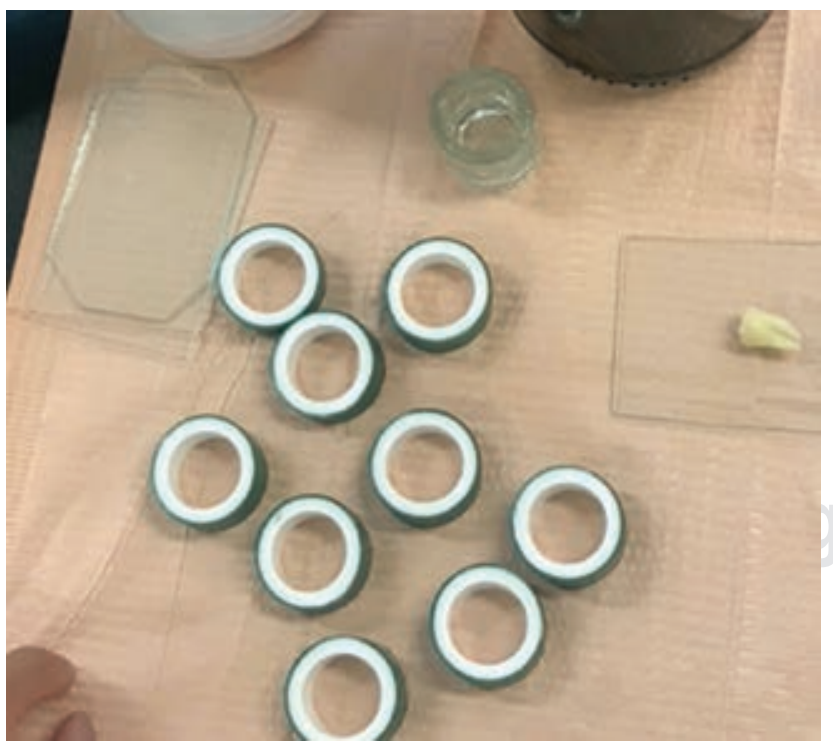

Figura 3: Montaje en acrílico.

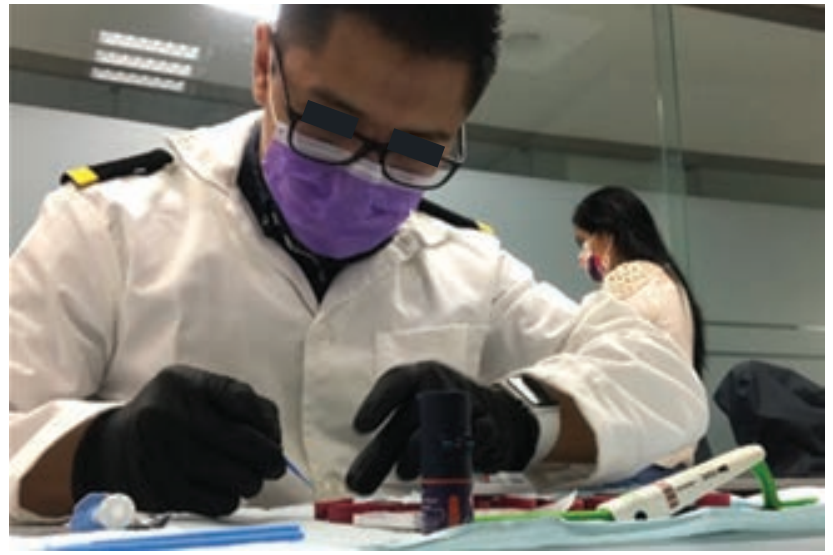

Figura 4: Aplicación de adhesivo Singl Bond Universal.

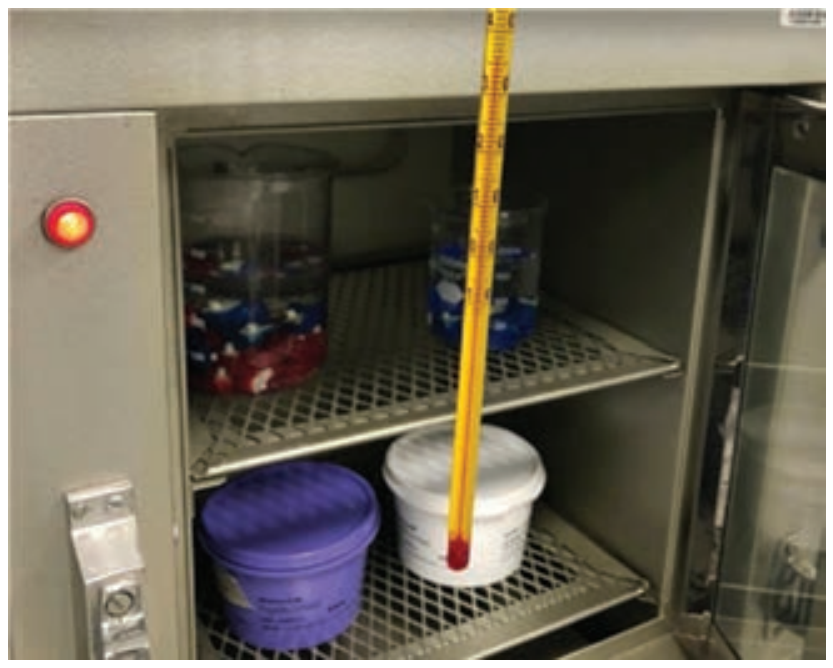

Figura 5: Muestras en el ambientador.

Se encontró que RelyX Ultimate tuvo mayor resistencia al cizallamiento con una media de 12.6444 en comparación con RelyX U200, con una media de 7.5569.

La prueba de Levene se utilizó para una prueba estadística inferencial para evaluar la igualdad de las varianzas.

En este caso fueron analizados dos grupos, cuando la significancia bilateral es distinta por la varianza, se refiere inmediatamente a la estadística de Levene; si ésta es mayor a 0.05 se toma la primera significancia bilateral. En este caso, la significancia de Levene es de 0.854 , por lo que se tomó la primera hilera de la significancia bilateral que es de 0.000 (Tabla 3).

Se realiza la prueba en t para la igualdad de medidas, con 95\% de intervalo de confianza en la diferencia. 
Los límites del intervalo de confianza para la diferencia nos indican que para la variable «Fuerza en MPa» los límites para los dos grupos están entre -5.785 y -4.389 puntos (Tabla 4).

En el histograma se puede observar la normalidad de los datos, mostrando la frecuencia estadística, la cual es el número de veces en el cual se repite la fuerza medida en MPa de dicha investigación.

La variable tiene una distribución aproximada a la normal, obteniendo una media de 10.10, la cual es el valor medio de la fuerza en MPa de dicha investigación, con desviación estándar de 2.792; el número total de casos es de 40 (Figura 7).

\section{DISCUSIÓN}

El conocimiento acerca de los cementos autograbado de resina, sus técnicas de aplicación y lo que implica cada una de ellas es fundamental para los dentistas, ya que va de por medio la salud de los pacientes. ${ }^{16,17}$

Desde su surgimiento en el año 2002, los cementos de resina proyectaron un sistema nuevo y sencillo logrando simplificar la multitud de los materiales utilizados en tratamientos realizados con anterioridad; sin embargo, el tratamiento previo del diente al utilizar un cemento de grabado total tiene una mayor eficiencia. ${ }^{18-21}$

El presente trabajo muestra la diferencia de resistencia a la adhesión de dos sistemas de cementación, observando

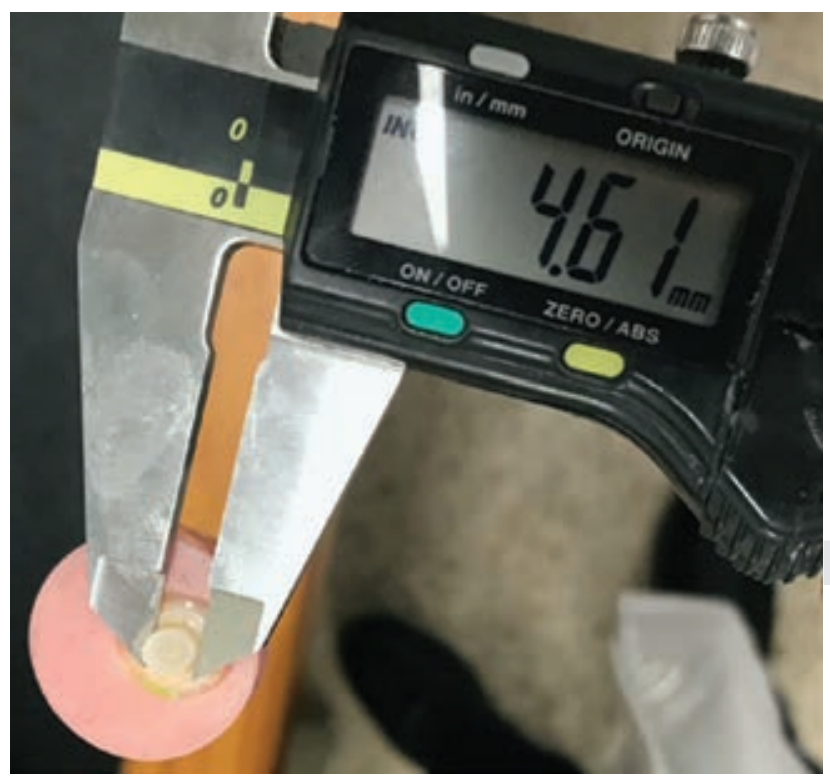

Figura 6: Medición del diámetro del botón.

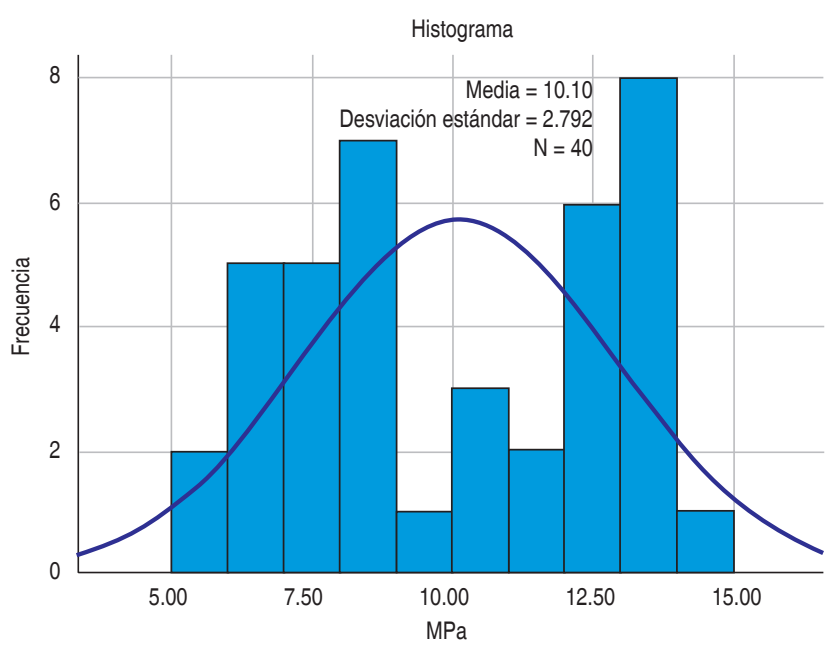

Figura 7: Frecuencia estadística. $\mathrm{MPa}=$ megapascales.

una diferencia en la fuerza de adhesión entre ambos cementos al momento de ser aplicada la fuerza de cizalla. ${ }^{22-24}$

En investigaciones anteriores se observa que el tratamiento previo de la dentina mejora la resistencia adhesiva. Diversos autores concluyeron que hubo diferencias significativas en la interface cemento-dentina cuando compararon un sistema autograbador con un sistema de grabado total, ${ }^{10}$ concluyeron que el cemento de grabado total crea entrelazados micromecánicos entre el material adhesivo y la dentina grabada mejorando la adhesión dentinaria. Por otra parte, Esclassan Noirrit ${ }^{11}$ comparó las técnicas de autograbado y grabado total en cuanto a la formación de capa híbrida. No hallaron diferencias significativas entre estas dos técnicas. Beher y colegas ${ }^{25}$ observaron que los cementos autoadhesivos presentan un grado de adhesión comparable con agentes cementantes convencionales como el cemento de silicato y el fosfato de zinc, no existe evidencia ni de desmineralización ni penetración a la dentina siendo, quizá, una de las razones la alta viscosidad de estos cementos y la rápida desactivación de los monómeros ácidos por la reacción ácido-base. Por otra parte, se ha observado que los cementos autoadhesivos tienen una menor acidez que el ácido fosfórico al 37\% y no consiguen un acondicionado tan profundo, ya sea en esmalte o en dentina, según Tay y Pashley. ${ }^{8} \mathrm{Al}$ analizar los resultados y con base en los antecedentes revisados con anterioridad, concluimos que nuestra investigación fue factible y llegamos a nuestro propósito, en el cual comprobamos la mayor adhesión de cementos de grabado total, los cuales son sometidos a un previo tratamiento del diente. ${ }^{21,26-28}$ 
Una de las grandes limitantes que se nos presentó en nuestra investigación fue la emergencia sanitaria ocurrida por el COVID-19, esto provocó modificar nuestro universo de estudio y modificar las fechas que ya se tenían contempladas para la realización de la prueba piloto y la fase experimental, así como la dificultad de llevar a cabo dichas pruebas en el laboratorio de materiales dentales de la Universidad Nacional Autónoma de México. Sin embargo, contamos siempre con el apoyo de nuestros asesores y director de tesis para realizar nuestro proyecto de investigación en las instalaciones de la Escuela Militar de Odontología, lo cual nos enorgullece, al saber que pese a los hechos ocurridos en nuestro país dejamos una puerta abierta a futuras generaciones para que realicen estudios en la máquina de ensayo universal Instron del laboratorio de nuestra grandiosa institución. Recomendamos que se incremente el número de muestras en futuros estudios y también la utilización de microscopia para evaluar el estado del sustrato al inicio y al final del experimento. ${ }^{29-32}$

\section{Tabla 1: Resultados obtenidos en la máquina Instron.}

Fuerza de adhesión al cizallamiento en megapascales

\begin{tabular}{cc}
\hline $\begin{array}{c}\text { Cemento de autograbado. } \\
\text { RelyX U200 Clicker 3M }{ }^{\mathrm{TM}}\end{array}$ & $\begin{array}{c}\text { Cemento de grabado total. } \\
\text { RelyX Ultimate Clicker 3M }\end{array}$ \\
\hline 9.61 & 13.35 \\
8.09 & 10.95 \\
8.48 & 13.44 \\
5.49 & 12.59 \\
5.94 & 12.93 \\
8.81 & 12.45 \\
6.79 & 13.18 \\
8.47 & 13.79 \\
8.89 & 13.53 \\
7.53 & 12.87 \\
6.51 & 12.39 \\
6.63 & 12.66 \\
7.41 & 13.21 \\
6.04 & 13.27 \\
7.73 & 10.15 \\
8.46 & 11.55 \\
8.19 & 13.48 \\
7.52 & 11.83 \\
7.59 & 14.39 \\
6.97 & 10.87 \\
\hline
\end{tabular}

Tabla 2: Estadísticas de grupo $(\mathrm{N}=40)$.

\begin{tabular}{lcc}
$\begin{array}{l}\text { Grupo } \\
\text { megapascales }\end{array}$ & $\begin{array}{c}\text { Media } \pm \text { desviación } \\
\text { estándar }\end{array}$ & $\begin{array}{c}\text { Desviación } \\
\text { error promedio }\end{array}$ \\
\hline $\begin{array}{l}\text { RelyX U200 } \\
\text { Clicker, } N=20 \\
\text { RelyX Ultimate } \\
\text { Clicker, N }=20\end{array}$ & $7.5569 \pm 1.09774$ & 0.24546 \\
\hline
\end{tabular}

Tabla 3: Prueba de muestras independientes.

\begin{tabular}{cc}
\multicolumn{2}{c}{ Megapascales } \\
\hline $\begin{array}{c}\text { Se asumen } \\
\text { varianzas iguales }\end{array} \quad \begin{array}{c}\text { No se asumen } \\
\text { varianzas iguales }\end{array}$
\end{tabular}

Prueba de Levene de igualdad de varianzas
$\mathrm{F}$
0.034
Significancia
0.854

Prueba $t$ de student para la igualdad de medias $\mathrm{t}$

Grados de libertad (GL)

Significancia bilateral

Diferencia de medias
$-14.751$

38

0.000

$-5.08750$
$-14.751$

37.994

0.000

$-5.08750$
Se recomienda dar continuación a este estudio, enfocándolo en el tratamiento previo de la dentina, con un número de especímenes más grande, para tener más certeza y apoyar nuestros resultados.

Asimismo, si existiesen cementos más actuales, estudiarlos en trabajos posteriores comparándolos con estas técnicas de cementación, con la finalidad de que en un futuro se adopte el uso de cementos de grabado total, dado que la dentina en su composición es más compleja que el esmalte, debido a su porcentaje de agua y tejido orgánico.

\section{CONCLUSIONES}

Una vez expuestos los resultados y discutidos los mismos, llegamos a las siguientes conclusiones:

1. La fuerza de adhesión del cemento RelyX U200 Clicker fue menor a la del cemento RelyX Ultimate Clicker de 3M. 
Tabla 4: Prueba de muestras independientes, intervalo de confianza al $95 \%$.

\begin{tabular}{lcc} 
& \multicolumn{2}{c}{ Megapascales } \\
\cline { 2 - 3 } Prueba t de Student para la igualdad de medias & Se asumen varianzas iguales & No se asumen varianzas iguales \\
\hline Diferencia de error estándar & 0.34490 & 0.34490 \\
$95 \%$ de intervalo de confianza de la diferencia & -5.78571 & -5.78571 \\
Inferior & -4.38929 & -4.38929 \\
Superior &
\end{tabular}

2. Se obtuvieron resultados favorables con el cemento RelyX Ultimate Clicker de $3 \mathrm{M}$, ya que su fuerza de adhesión a la dentina es mayor que la del cemento RelyX U200 Clicker.

3. Tras las pruebas realizadas, se puede evidenciar que existe diferencia estadísticamente significativa en la resistencia adhesiva entre el cemento de resina RelyX U200 Clicker de 3M y el cemento RelyX Ultimate de $3 \mathrm{M}$ a dentina en dientes extraídos previamente.

4. Al analizar los resultados obtenidos, se concluye que a pesar de que los cementos de grabado total tienen un mayor costo en comparación con los cementos de autograbado, el beneficio a los tratamientos indirectos es de mayor calidad y durabilidad.

5. El protocolo de cementación de cementos de grabado total conlleva un mayor número de pasos a comparación de los cementos de autograbado, pero el beneficio y calidad del trabajo sigue siendo de mejor calidad, ya que, al aplicar el grabado total a dentina, las restauraciones tendrán no sólo una adhesión química, sino también una adhesión mecánica al abrir los túbulos dentinarios.

6. Los cementos de grabado total siguen siendo la mejor opción para tratamientos odontológicos indirectos, ya que éstos han ido evolucionando al paso del tiempo para su mayor durabilidad y biocompatibilidad en el diente tratado.

7. Se concluye que los cementos de grabado total utilizados en la clínica dental de la SEDENA brindan un mayor beneficio para los pacientes como para la institución, ya que con ello se tendrán restauraciones de mejor calidad y durabilidad, evitando el desprendimiento de las restauraciones, con ello se evita la reincidencia del tratamiento, dando sólo tratamientos preventivos y de revisión para mantener las restauraciones y la buena salud bucodental de los pacientes.

\section{REFERENCIAS}

1. Gwinnett AJ, Matsui A. A study of enamel adhesives. The physical relationship between enamel and adhesive. Arch Oral Biol. 1967; 12 (12): 1615-20.

2. Buonocore MG. New anterior restorative materials. Int Dent J. 1968; 18 (2): 406-20.

3. Falconí-Borja GM, Molina-Pule CG, Velásquez-Ron BV, Armas-Vega AC. Evaluación del grado de microfiltración en restauraciones de resina compuesta, comparando dos sistemas adhesivos tras diferentes períodos de envejecimiento. Rev Fac Odontol Univ Antioq. 2016; 27 (2): 281-295.

4. Takamizawa T, Barkmeier WW, Tsujimoto A, Berry TP, Watanabe $\mathrm{H}$, Erickson RL et al. Influence of different etching modes on bond strength and fatigue strength to dentin using universal adhesive systems. Dent Mater. 2016; 32 (2): e9-e21. doi: 10.1016/j. dental.2015.11.005.

5. Machi R. Materiales dentales. 4a ed. Buenos Aires, Argentina: Editorial Panamericana; 2007.

6. Behr M, Rosentritt M, Regnet T, Lang R, Handel G. Marginal adaptation in dentin of a self-adhesive universal resin cement compared with well-tried systems. Dent Mater. 2004; 20 (2): 191197. doi: 10.1016/s0109-5641(03)00091-5.

7. Miguelena Muro KE, Guerrero Ibarra J, Garcilazo Gómez A, Ríos Szalay E. Análisis de resistencia al desplazamiento de dos cementos de resina, en dentina intrarradicular. Rev Odontol Mex. 2016; 20 (4): $238-242$.

8. Pashley DH, Tay FR. Aggressiveness of contemporary selfetching adhesives. Part II: etching effects on unground enamel. Dent Mater. 2001; 17 (5): 430-444. doi: 10.1016/s01095641(00)00104-4.

9. Bitter K, Schubert A, Neumann K, Blunck U, Sterzenbach G, Rüttermann $\mathrm{S}$. Are self-adhesive resin cements suitable as core build-up materials? Analyses of maximum load capability, margin integrity, and physical properties. Clin Oral Investig. 2016; 20 (6): 1337-45.

10. Sridhara KS, Mankar S, Jayshankar CM, Vinaya K. Scanning electron microscopic study of teeth restored with fiber posts and composite resin: An in vitro study. J Pharm Bioallied Sci. 2014; 6 (Suppl 1): S74-9.

11. Esclassan NE, Grégoire G, Cournot M. Morphological study of fiber-reinforced post-bonding system-root dentin interface by evaluation of two bonding systems. J Dent. 2008; 36 (3): 204-13

12. Chávez-Lozada J, Urquía-Morales C. In-vitro evaluation of bond strength of four self-etching cements. Acta Odontol Latinoam. 2017; 30 (3): 101-108. 
13. Vásquez Domínguez LJ, Arreola Martínez G, Larriva Loyola J, Rodríguez Ciódaro A, Güiza Cristancho EH. Medición de la capa híbrida resultante del uso de cementos autograbadores de uno y dos pasos. Universitas Odontológica. 2018; 37. Disponible en: https://doi.org/10.11144/Javeriana.uo37-78.mchr

14. Valdés Álvarez A. Adhesión dentinaria: fundamento, evolución y perspectiva. Gaceta Dental. 2019. Disponible en: https:// gacetadental.com/2019/06/adhesion-dentinaria-fundamenteevolucion-y-prespectiva-89137/

15. España Tost J, Berini Aytes L, Espaza de Desa E. Estudio comparativo de resina preparada. 2018. Diposit.ub.edu

16. Garcilazo-Gómez A, Miguelena-Muro KE, Guerrero-lbarra J, RiosSzalay E, Bonilla-Haro R. Factores que afectan y mejoran la adhesión en dentina, una puesta al día. Una revisión de la literatura. Rev ADM. 2019; 76 (3): 162-168.

17. Cruz CG, Vázquez REM. Resistencia al cizallamiento utilizando adhesivo de grabado total y autograbante con y sin hipoclorito de sodio en dentina. Rev ADM. 2017; 74 (5): 224-230.

18. Roedel L, Bednarzig V, Belli R, Petschelt A, Lohbauer U, Zorzin J. Self-adhesive resin cements: $\mathrm{pH}$-neutralization, hydrophilicity, and hygroscopic expansion stress. Clin Oral Investig. 2017; 21 (5): 1735-1741. doi: 10.1007/s00784-016-1947-4.

19. Tartari T, Wichnieski C, Bachmann L, Jafelicci M Jr, Silva RM, Letra A et al. Effect of the combination of several irrigants on dentine surface properties, adsorption of chlorhexidine and adhesion of microorganisms to dentine. Int Endod J. 2018; 51 (12): 1420-1433. doi: 10.1111/iej.12960.

20. Okulus Z, Buchwald T, Czarnecka B, Voelkel A. The effect of bonding system application on surface characteristics of bovine dentin and enamel. Mater Sci Eng C Mater Biol Appl. 2017; 76: 1224-1231. doi: 10.1016/j.msec.2017.03.205.

21. Carrillo SC. Dentina y adhesivos dentinarios. Conceptos actuales. Rev ADM. 2006; 63 (2): 45-51.

22. Lang-Salas MG, Villarreal-Romero LA, Domínguez-Monreal JA, Cuevas-González JC, Donohué-Cornejo A, Reyes-López SY et al. Evaluación de la adhesión de sistemas adhesivos de grabado total en esmalte dental bovino usando un agente desproteinizante: un estudio in vitro. Rev ADM. 2020; 77 (1): 22-27.

23. Martín Hernández J. Aspectos prácticos de la adhesión a dentina. Av Odontoestomatol. 2004; 20 (1): 19-32.

24. Manso AP, Carvalho RM. Dental cements for luting and bonding restorations: self-adhesive resin cements. Dent Clin North Am. 2017; 61 (4): 821-834. doi: 10.1016/j.cden.2017.06.006.

25. Weiser F, Behr M. Self-Adhesive Resin Cements: A Clinical Review. Journal of Prosthodontics, 2015; 24: 100-108.

26. Kumari RV, Poluri RK, Nagaraj H, Siddaruju K. Comparative evaluation of bond strength of dual-cured resin cements: an in-vitro study. J Int Oral Health. 2015; 7 (Suppl 1): 43-47.

27. Frassetto A, Breschi L, Turco G, Marchesi G, Di Lenarda R, Tay FR et al. Mechanisms of degradation of the hybrid layer in adhesive dentistry and therapeutic agents to improve bond durability-A literature review. Dent Mater. 2016; 32 (2): e41-e53. doi: 10.1016/j.dental.2015.11.007.

28. Mandri MN, Aguirre Grabre de Prieto A, Zamudio ME. Sistemas adhesivos en odontología restauradora. Odontoestomatología. 2015; 17 (26): 50-56.

29. Lombardo N, Cagnone G, Casadoumecq A. Facultad de Odontología Posgrado. Buenos Aires: UBA; 2019.

30. Arola DD, Gao S, Zhang H, Masri R. The tooth: its structure and properties. Dent Clin North Am. 2017; 61 (4): 651-668. doi: 10.1016/j.cden.2017.05.001.

31. Jalali H, Farid F, Kulivand S, Nokar S, Dadgar K. Effect of different irrigants applied after post space preparation on push-out bond strength of a self-etch resin cement. J Dent (Tehran). 2018; 15 (4): 222-229.

32. Tong Z, Zhang Y, Wei X. The effect of human serum and dentin powder alone or in combination on the antibacterial activity of sodium hypochlorite against Enterococcus faecalis. Arch Oral Biol. 2019; 97: 72-76. doi: 10.1016/j.archoralbio.2018.10.008.

\section{Correspondencia:}

Rogelio Moreno Landa

E-mail: roger.moreno1089|@gmail.com 